\title{
Spatially Resolved Ballistic Optoelectronic Transport Measured by Quantized Photocurrent Spectroscopy
}

\author{
Klaus-Dieter Hof, ${ }^{\dagger}$ Franz J. Kaiser, ${ }^{\ddagger}$ Markus Stallhofer, ${ }^{\S}$ Dieter Schuh, ${ }^{\prime}$ Werner Wegscheider, ${ }^{\perp}$ \\ Peter Hänggi, ${ }^{\ddagger}$ Sigmund Kohler, ${ }^{\ddagger}, \#$ Jörg P. Kotthaus, ${ }^{\dagger}$ and Alexander W. Holleitner ${ }^{*}, \S$ \\ ${ }^{\dagger}$ Fakultät für Physik and Center for NanoScience (CeNS), Ludwig-Maximilians-Universität, \\ Geschwister-Scholl-Platz 1, D-80539 München, Germany, ${ }^{*}$ Institut für Physik, Universität Augsburg, \\ Universitätsstrasse 1, D-86135 Augsburg, Germany, ${ }^{\S}$ Walter Schottky Institut and Physik Department, \\ Technische Universität München, Am Coulombwall 3, D-85748 Garching, Germany, "Institut für Experimentelle \\ und Angewandte Physik, Universität Regensburg, D-93040 Regensburg, Germany, ${ }^{\perp}$ Laboratorium für \\ Festkörperphysik, HPF E 7, ETH Zürich, Schafmattstrasse 16, 8093 Zürich, Switzerland, and "Instituto de Ciencia de \\ Materiales de Madrid, CSIC, E-28049 Madrid, Spain
}

\begin{abstract}
GaAs-based quantum point contacts (QPCs) are exploited to spatially resolve and analyze the ballistic, nonequilibrium flow of photogenerated electrons in a nanoscale circuit. Electron-hole pairs are photogenerated in a two-dimensional electron gas (2DEG), and the resulting current through an adjacent QPC is measured as a function of the laser spot position. The transmission of photogenerated electrons through the QPC is governed by the energy dispersion and the quantized momentum values of the electron modes in the QPC
\end{abstract}

KEYWORDS Ballistic optoelectronic quantum transport, nanoscale electronics

$\longrightarrow$ uantum point contacts (QPCs) are one-dimensional (1D) constrictions in electronic circuits, and they exhibit a conductance quantized in steps of $2 e^{2} / h$. The discovery of QPCs by van Wees ${ }^{1}$ and Wharam ${ }^{2}$ initiated a large number of experiments in nanoscale circuits. QPCS have been utilized as electronic beam splitters in electron interference experiments, ${ }^{3-6}$ and they have been exploited in very sensitive detection schemes to quantify single charge and spin states in nanoscale circuits. ${ }^{7-12}$ Further, recent experimental work based on QPCs has succeeded in imaging the coherent electron flow at the Fermi-energy $E_{\text {Fermi }}$ of highmobility, two-dimensional electron gases (2DEGs). ${ }^{13-15}$ In such 2DEGs, the ballistic mean free path is much greater than the Fermi wavelength, and electrons can coherently propagate for several micrometers. ${ }^{14,15}$ Here, we demonstrate the use of GaAs-based QPCs to explore the nonequilibrium dynamics of photogenerated charge carriers in nanoscale circuits. We measure the optical beam induced current $(\mathrm{OBIC})^{16}$ through a QPC, when charge carriers are optically created in an adjacent 2DEG. We demonstrate that such an experimental scheme is sensitive enough to detect and characterize the flow patterns of photogenerated charge carriers in nanoscale circuits. By analyzing OBIC maps of the circuits, we find that photogenerated electrons are elastically reflected at the side walls of the circuits and that photoge-

* Corresponding author. E-mail: holleitner@wsi.tum.de. Received for review: 03/11/2010

Published on Web: 09/20/2010 nerated electrons propagate ballistically in nanoscale circuits alike "billiard balls". ${ }^{17}$ So far, ballistic photocurrents have been generated and detected only by optical means by a coherent phase control of two or more laser beams. ${ }^{18-20}$ There, indications for ballistic lateral photocurrents with length scales of a few tens to hundreds of nanometers have been detected. We find that a ballistic optoelectronic transport can occur across several micrometers. We further demonstrate that the presented technique allows analyzing the laser-induced current in energy. Therefore, the technique could prove useful as a characterization method to resolve the energy, momentum, and even spin distribution of photoexcited electrons in nonlinear electron devices, including nanoscale photodetectors and fast transistors, such as high electron mobility transistors (HEMTs) and spintronic devices.

The QPCs sketched in Figure 1a are lithographically defined by a shallow etch technique ${ }^{11}$ in a GaAs quantum well structure, which contains a 2DEG. Furthermore, the QPC is supplemented with an opaque topgate (see ref 21 and Supporting Information). The QPC is placed between two sections of the 2DEG, which acts as source and drain reservoirs. The measured conductance quantization of such QPCs in steps of $2 e^{2} / h$ is shown in Figure $1 \mathrm{~b} .{ }^{1,2}$ Each step reflects a $1 \mathrm{D}$ subband of the QPC. In the presented experiments, we scan a laser spot across the 2DEG reservoirs and simultaneously measure the OBIC through the QPC. Figure 2 a shows a micrograph of the circuit, and Figure $2 \mathrm{~b}$ depicts the corresponding OBIC map of the circuit as a function of 


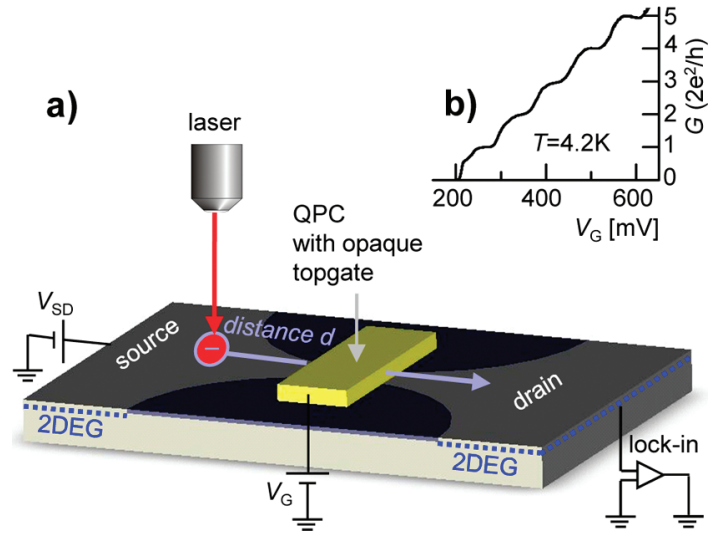

FIGURE 1. OBIC mapping of optoelectronic circuits. (a) Schematic diagram showing a QPC with an opaque topgate (yellow) and optical excitation spot (red). (b) Dark conductance of the QPC as a function of the topgate voltage $V_{\mathrm{G}}$.

a)
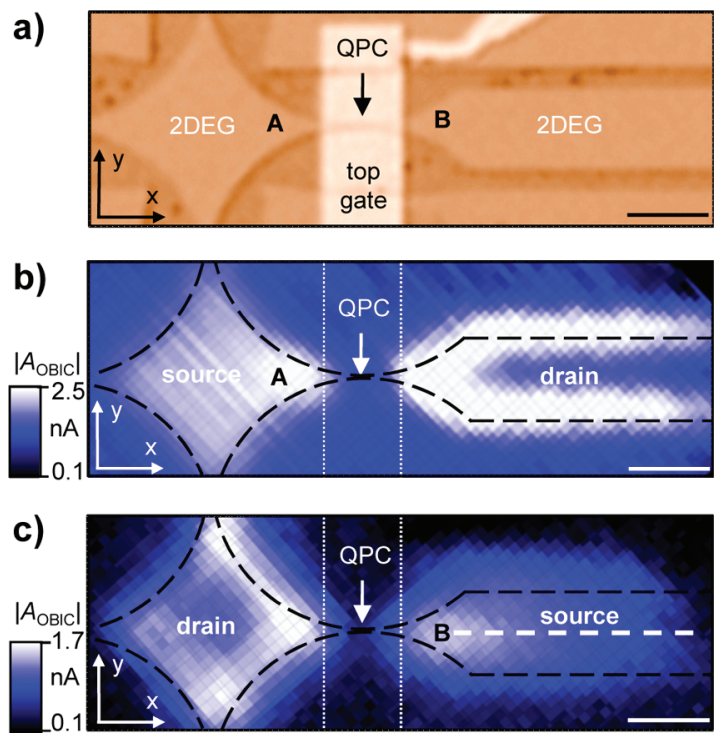

FIGURE 2. (a) Micrograph of a circuit with a QPC below a topgate, positioned between two sections of a 2DEG. (b) OBIC map of the circuit in (a) at a trigger frequency of $76 \mathrm{MHz}$ when left (right) 2DEG section acts as source (drain) reservoir $\left(V_{\mathrm{SD}}=-2 \mathrm{mV}, V_{\mathrm{G}}=330 \mathrm{mV}\right.$, $T=3.3 \mathrm{~K}, E_{\text {Photon }}=1.552 \mathrm{eV}$, and $P_{\text {Laser }}=0.8 \mu \mathrm{W}$ ). (c) OBIC map when right (left) 2DEG section acts as source (drain) reservoir $\left(V_{\mathrm{SD}}\right.$ $=-2 \mathrm{mV}, V_{\mathrm{G}}=330 \mathrm{mV}, T=3.3 \mathrm{~K}, E_{\text {Photon }}=1.552 \mathrm{eV}$, and $P_{\text {Laser }}=$ $1 \mu \mathrm{W})$. Scale bars in $(\mathrm{a}-\mathrm{c})$ are $10 \mu \mathrm{m}$. Dashed lines in (b) and (c) show the boundaries of the circuits in (a) as a guide to the eye.

the spatial coordinates $x$ and $y$ at $4.2 \mathrm{~K}$. On the source reservoir, the signal exhibits a lobe structure pointing away from the QPC along the direction $-x$ with a maximum at position $A$. As will be discussed below, we interpret this lobe structure to originate from ballistic trajectories of photogenerated electrons. The photogenerated holes can give rise to a photoconductive gain effect. ${ }^{21-23}$ It dominates the OBIC on the etched boundaries of the drain reservoir but not on the source reservoir at the trigger frequency of $76 \mathrm{MHz}$, which is utilized as a reference frequency in the lock-in detection scheme (see Supporting Information). Switching the source and drain reservoirs, we detect the ballistic
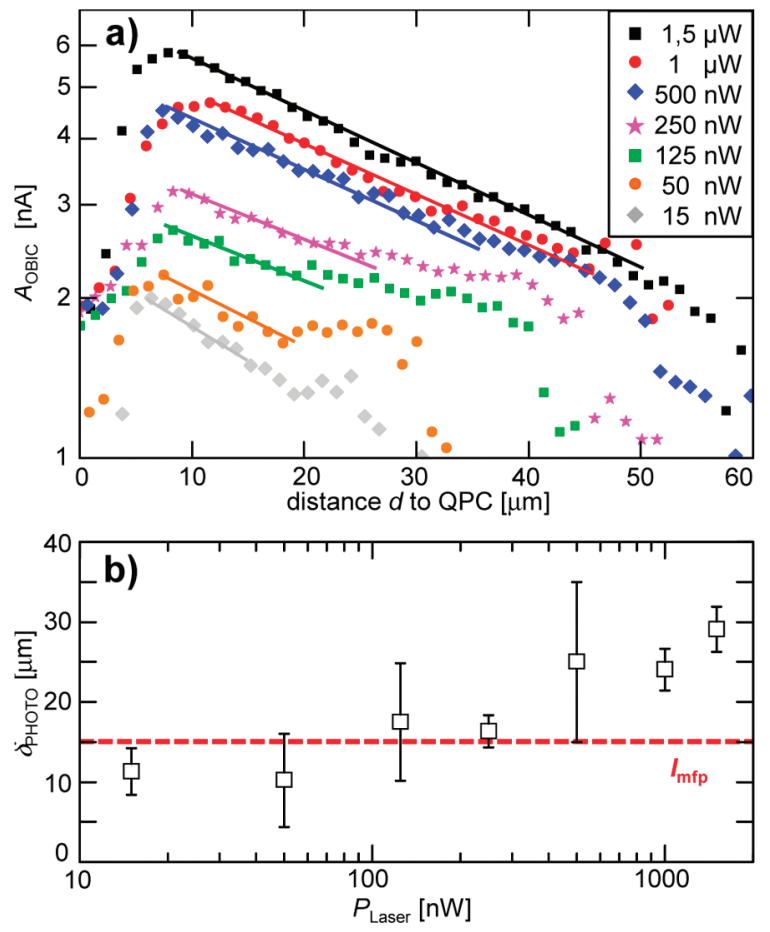

FIGURE 3. (a) Amplitude of the OBIC at position $B$ in Figure 2c along the dashed line for increasing $P_{\text {Laser }}$. The data are fitted by $\exp (-x \mid$ $\left.\delta_{\text {Pното }}\right)+\left|A_{\text {OFFSET }}\right|$, with an offset value $\left|A_{\text {OFFSET }}\right|$ (see Supporting Information). (b) Fitted values of $\delta_{\text {Рното as a function of } P_{\text {Laser }}}$ compared to the mean free path $l_{\mathrm{mfp}}$ in the 2DEG at $4.2 \mathrm{~K}$ (dashed line).

photocurrent lobe on the right-hand section of the 2DEG (Figure 2c). Again on the drain reservoir, the boundaries of the circuit appear in the OBIC map.

Salient advantages of the OBIC technique include that the intrinsic length scale of the photocurrent can be quantified. Figure 3 a shows the OBIC amplitude $A_{\mathrm{OBIC}}$ along the white dashed line in Figure 2c. For all laser powers $P_{\text {Laser }}$, the signal decays exponentially as a function of the distance $d$ to the QPC. The typical decay length $\delta_{\text {Photon }}$ is in the range of several micrometers (Figure $3 \mathrm{~b}$ ), and it is comparable to the electron mean free path $l_{\mathrm{mfp}}=15.1 \mu \mathrm{m}$ of the $2 \mathrm{DEG}$ at $4.2 \mathrm{~K}$. Values of $\delta_{\text {Photon }}$ on the micrometer scale are striking, since so far, ballistic lateral photocurrents with length scales of only a few tens to hundreds of nm have been indirectly detected by optical means. ${ }^{18-20}$ Here, we directly detect the photogenerated electrons by sensing the ballistic photocurrent through a QPC. For large $P_{\text {Laser, }}$, we observe that $\delta_{\text {Photon }}$ slightly exceeds $l_{\mathrm{mfp}}$, which possibly stems from a partial population of the second subband of the 2DEG at large laser powers ${ }^{24}$ (see Supporting Information).

We compute the OBIC map from a Monte Carlo simulation of classically propagating, noninteracting electrons in a flat potential with hard walls (Figure $4 \mathrm{a}$ and b). In our model, an electron excited at position $(x, y)$ in the source reservoir contributes to the OBIC if it reaches the drain. Thus, the OBIC map is proportional to the arrival probability $w(x, y)$. The initial propagation direction is random. When an elec- 


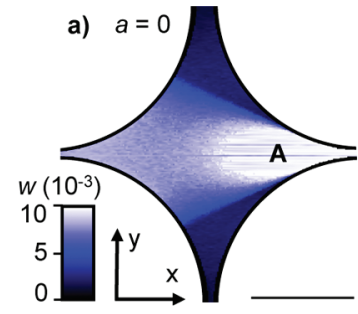

b) $a=0$
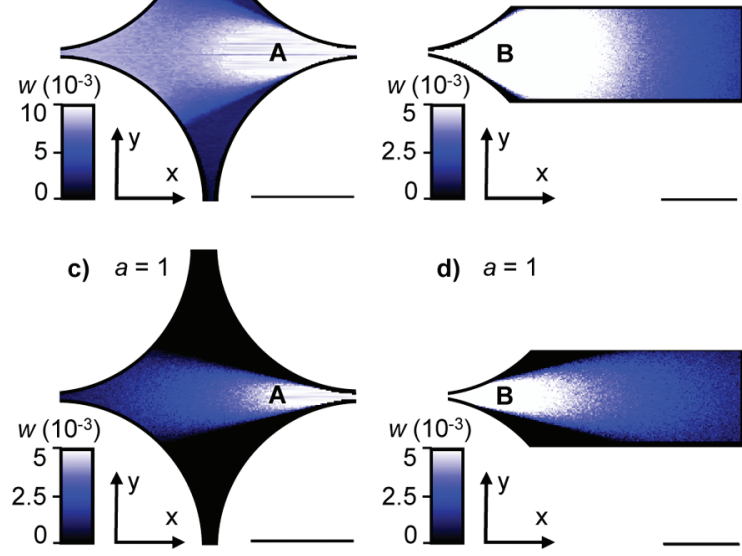

d) $a=1$

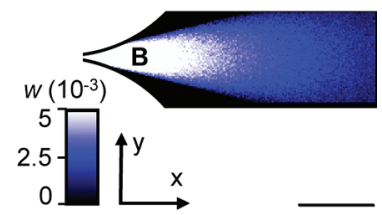

FIGURE 4. (a) This figure shows simulations of the electron flow within the left rhombic 2DEG section of the optoelectronic circuit as in Figure $2 \mathrm{a}$ for a boundary absorption probability $a=0$ and in (c) for $a=1$. (b) Computed electron flow in the right 2DEG section of the circuit in Figure $2 \mathrm{a}$ for $a=0$ and in (d) for $a=1$. Scale bars are $10 \mu \mathrm{m}$.

tron hits a wall, it is reflected elastically unless it is absorbed with probability $a$. Since the size of the sample is of the same order as $l_{\mathrm{mfp}}$, we have to consider the momentum scattering of the electrons. Therefore, we weight the contribution of a path with length $l$ with the factor $\exp \left(-l / l_{\mathrm{mfp}}\right)$, which is consistent with the findings in Figure $3 \mathrm{~b}$. The simulation with boundary absorption probability $a=0$ (Figure $4 \mathrm{a}$ and $\mathrm{b}$ ) largely reproduces the OBIC map of the respective source reservoirs of Figure $2 \mathrm{~b}$ and $\mathrm{c}$. Absorbing walls, i.e., $a=1$, lead to "dark areas" (Figure 4c and d) which are not observed in the experiment. Thus, we tentatively conclude that the optoelectronic circuit in Figure 2 a embodies a classical electron billiard in which photogenerated electrons are reflected several times before they reach the QPC. The geometrical apertures close to the QPC favors trajectories with $k$-vectors alongside the $x$-direction. The latter finding is consistent with quantum simulations and measurements of the electron flux density in the vicinity of QPCs. ${ }^{13-15}$ However, we note that also at the trigger frequency of 76 $\mathrm{MHz}$, a small reminiscent signal of the photoconductive gain effect still superimposes the ballistic trajectories on the source reservoir (see Supporting Information).

In the present optoelectronic experiment, the electrons are optically created above the Fermi energy $E_{\text {Fermi }}$ of the 2DEG. The initial energy distribution of the photogenerated electrons in our circuits can be assumed to be Gaussian with a full width at half-maximum (FWHM) $\sim 12 \mathrm{meV}$ (see Supporting Information). The average transit time $\tau_{\text {transit }}$ of a photogenerated electron from the laser spot to the QPC can be estimated to be $\tau_{\text {transit }} \approx l / \nu_{\text {Fermi }} \approx l_{\mathrm{mfp}} / \nu_{\text {Fermi }}=15.1 \mu \mathrm{m} /$ $2.3 \times 10^{5} \mathrm{~ms}^{-1} \approx 66 \mathrm{ps}$, with $v_{\text {Fermi }}$ the Fermi velocity. Previous work demonstrated, ${ }^{25-27}$ that at an excess energy $\Delta=E-E_{\text {Fermi }}$ of only a few meV above $E_{\text {Fermi }}$, the electron trajectories in 2DEGs are dominated by electron-electron scattering. The electron-electron scattering time in 2DEGs is indirectly proportional to $\Delta^{2} \ln \Delta .^{25,26}$ For $\Delta \sim \mathrm{meV}$, the electron-electron scattering time is in the range of a few ps. ${ }^{27}$ Hence, photogenerated electrons can be assumed to scatter at a few electrons during $\tau_{\text {transit }}$ before they reach the QPC. ${ }^{28}$ Following the work by D. Snoke et al., ${ }^{29}$ hereby, the energy distribution of photogenerated electrons approaching the QPC can be estimated to be a combination of a Gaussian and a Maxwell-Boltzmann distribution. This raises the question, whether the resulting energy distribution of the photogenerated electrons approaching the QPC is narrow enough such that the subbands of the QPC can be resolved in the OBIC.

All measurements in Figure 5 are detected at position $B$, as in Figure 2c. As depicted in Figures $5 \mathrm{a}-\mathrm{c}$, the OBIC exhibits steps (see triangles) as a function of $V_{\mathrm{G}}$ for a photon energy $E_{\text {photon }}$ larger than the interband energy of the quantum well $E_{\mathrm{QW}}=1.545 \mathrm{eV} .{ }^{21}$ We interpret the steps to reflect the subbands of the QPC. ${ }^{1,2}$ The step amplitude is strongly influenced by the impedance of the circuits, which varies with the number of photogenerated charge carriers and $V_{\mathrm{G}}{ }^{21}$ Since we detect steps, we conclude that the energy distribution of photogenerated electrons approaching the QPC is narrower than the subband spacing of the QPC. In nonlinear conductance measurements without laser, we determine the subband spacing of the particular QPC to be $\sim 4 \mathrm{meV}$. For $E_{\mathrm{Photon}}<E_{\mathrm{QW}}$, the laser does not create electronhole pairs in the source reservoir. Therefore, we interpret the signal in Figure $5 d$ to originate from a capacitive crosscoupling between the source and the drain reservoirs (see Supporting Information).

In a simple model, we assume that photogenerated electrons approaching the QPC have an energy value $E^{\mathrm{OPT}}$ $\geq E_{\mathrm{Fermi}}$ and a $k$-vector $k^{\mathrm{OPT}}$ parallel to the $x$-direction (Figure $5 \mathrm{e})$. In the $\mathrm{QPC}$, the $k$-vector along the $y$-direction has quantized values $\pm n \cdot \pi / W$, with $n=1,2, \ldots$ and $W$ the width of the QPC. ${ }^{30}$ Hereby, the $n$-th subband in the QPC has an energy $E_{n}^{\mathrm{QPC}}=\left(\hbar^{2} \pi^{2} n^{2}\right) /\left(2 m^{*} W^{2}\right)+\left(\hbar^{2} k_{x}{ }^{2}\right) /\left(2 m^{*}\right)$, with $\hbar$ the Planck's constant, $m^{*}$ the effective electron mass, and $k_{x}$ the $k$-vector within the QPC along the $x$-direction. The photogenerated electrons can propagate through the QPC when $\left(E^{\mathrm{OPT}}, k^{\mathrm{OPT}}\right)$ equals the values of the electron modes within the QPC (blue circle in Figure $5 \mathrm{e})$. For $\left(E^{\mathrm{OPT}}, k^{\mathrm{OPT}}\right) \neq\left(E^{\mathrm{QPC}}\right.$, $\left.k^{\mathrm{QPC}}\right)$, one expects that electron-electron scattering events level out the energy and the momentum mismatch during the adiabatic coupling of the photogenerated electrons into the QPC. ${ }^{28}$

The optoelectronic circuit allows considering a situation with no thermalized electrons flowing from drain to source but with photogenerated electrons impinging on the source side of the QPC (Figure $5 f$ and g). Hereby, large angle electron-electron scattering events are reduced for the photogenerated electrons in the direct vicinity of the QPC. ${ }^{13-15}$ In turn, one expects that the OBIC is reduced, because a possible energy and momentum mismatch can- 

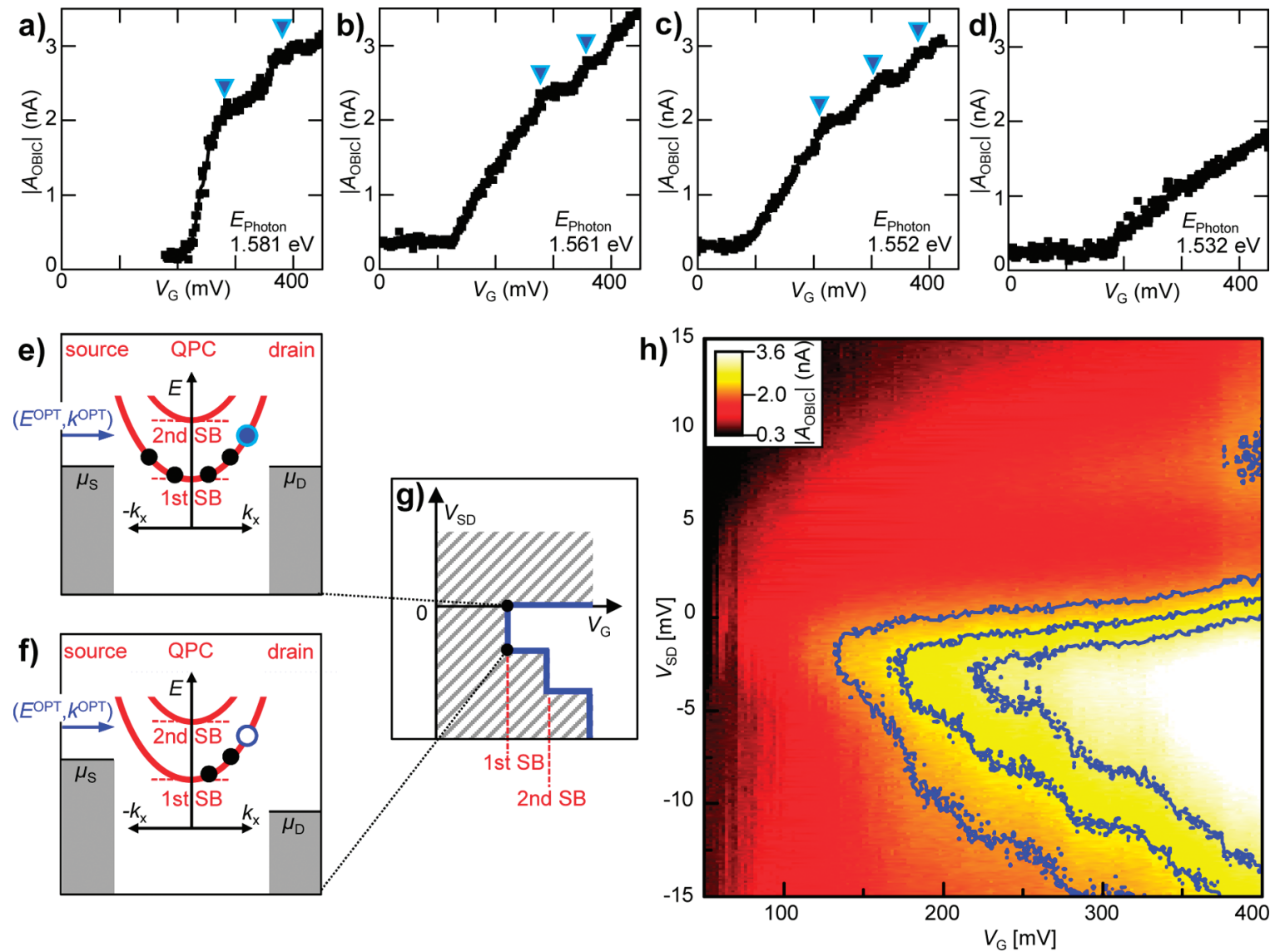

FIGURE 5. Nonlinear photocurrent spectroscopy on the QPC. (a-c) The OBIC at position $B$ as in Figure $2 \mathrm{c}$ at $3.4 \mathrm{~K}$ plotted as a function of $V_{\mathrm{G}}$ at a bias voltage $V_{\mathrm{SD}}=-5 \mathrm{meV}$ and $P_{\mathrm{LASER}}=50 \mathrm{nW}$. For $E_{\mathrm{Photon}} \geq E_{\mathrm{OW}}=1.545 \mathrm{eV}$, the signal exhibits steps (see triangles). (d) For $E_{\mathrm{Photon}}<E_{\mathrm{OW}}$, no steps are detected. $(\mathrm{e}-\mathrm{g})$ Model depicting the average energy and momentum $\left(E_{\mathrm{OPT}}, k_{\mathrm{OPT}}\right)$ of photogenerated electrons approaching the QPC at the source reservoir for (e) 0 and (f) negative bias $V_{\mathrm{SD}}=\mu_{\mathrm{D}}-\mu_{\mathrm{S}}$ (h) OBIC amplitude $\left|A_{\mathrm{OBIC}}\right|$ at position $B$ as in Figure $2 \mathrm{c}$ as a function of $V_{\mathrm{SD}}$ and $V_{\mathrm{G}}$ at $3.4 \mathrm{~K}, E_{\mathrm{Photon}}=1.552 \mathrm{eV}$, and $P_{\text {Laser }}=250 \mathrm{nW}$.

not be balanced. To demonstrate this effect, we measure the OBIC as a function of $V_{\mathrm{SD}}$ and $V_{\mathrm{G}}$ (Figure $5 \mathrm{~h}$ ). We note the following two features: First, the OBIC is strongly suppressed for $V_{\mathrm{SD}}>0$, when the QPC acts as a potential barrier to the photogenerated electrons created in the source reservoir $\left(V_{\mathrm{SD}}\right.$ $=\mu_{\mathrm{D}}-\mu_{\mathrm{S}}>0$, with $\mu_{\mathrm{S} / \mathrm{D}}$ the chemical potentials of the source and drain reservoirs) (see Supporting Information). Second, for $V_{\mathrm{SD}}<0$, the OBIC is reduced in steps of roughly the subband spacing of the QPC $\sim 4 \mathrm{meV}$. Hereby, the QPC acts as a scatterer for the photogenerated electrons in the source reservoir (white circle in Figure 5f), when no thermalized electrons flow from drain to source. In other words, then, a possible mismatch in energy and momentum between the photogenerated electrons and the 1D modes of the QPC cannot be leveled out by electron-electron scattering during the adiabatic coupling of the photogenerated electrons into the QPC. ${ }^{28}$ We note that the steps, which are marked as blue lines in Figure 5h, are consistent with the photocurrent steps in Figure $5 \mathrm{c}$ (blue triangle). We further point out that, strictly speaking, the notion of a Fermi energy deduced from the dark carrier concentration is no longer applicable for the photoexcited reservoirs $\left(\mu_{\mathrm{S}}\right.$ in the schemes of Figure $5 \mathrm{e}$ and f). However, an upper limit of the FWHM of a new quasiFermi level can be experimentally determined to be $\sim 3 \mathrm{meV}$ (see Supporting Information). Most importantly, this energy is less than the subband spacing of the $\mathrm{QPC} \sim 4 \mathrm{meV}$, and the above arguments are valid. In addition, if the photogenerated electrons would propagate through the QPC at the photoexcited energy without any energy relaxation during the propagation to the QPC, one expects that the $V_{\mathrm{G}}-V_{\mathrm{SD}}$ graph in Figure 5 h would show a broad maximum at a finite value $e V_{\mathrm{SD}} \neq 0$ and a FWHM of $\sim 12 \mathrm{meV}$. However, we do not detect such a broad peak. Instead, we resolve steps in the photocurrent with an energy spacing of $\sim 4 \mathrm{meV}$.

In summary, the OBIC technique applied to nanoscale circuits with QPCs is a powerful new tool for directly imaging the ballistic flow of photogenerated electrons in nanoscale circuits. We demonstrate that photogenerated electrons propagate ballistically in nanoscale circuits alike "billiard balls". The presented technique could prove useful as a characterization method to spatially resolve the charge, energy, and spin distribution of photogenerated electrons in nonlinear electron devices, including photodetectors, fast transistors, and graphene based devices. 
Acknowledgment. We thank S. Manus and M. Kroner for technical support. In addition, we gratefully acknowledge financial support from BMBF via nanoQUIT, the DFG Grant no. Ho 3324/4, the Center for NanoScience (CeNS), the German excellence initiative via the "Nanosystems Initiative Munich (NIM)" and "LMUexcellent".

Supporting Information Available. Details on the heterostructure, the sample preparation, the experimental technique, the offset values $A_{\text {offset }}$ of the exponential curves in Figure $3 a$, further details regarding the Monte Carlo simulation presented in Figure 4, a discussion of the photoconductive gain effect which is dominated by the photogenerated holes, a discussion of the capacitive cross-coupling between the source and drain contacts, and further control experiments. This material is available free of charge via the Internet at http://pubs.acs.org

\section{REFERENCES AND NOTES}

(1) VanWees, B. J.; et al. Phys. Rev. Lett. 1988, 60, 848.

(2) Wharam, D. A.; et al. J. Phys. C: Solid State Phys. 1988, 21, L209

(3) Neder, I.; Ofek, N.; Chung, Y.; Heiblum, M.; Mahalu, D.; Umansky, V. Nature 2007, 448, 333

(4) Litvin, L. V.; Tranitz, H.-P.; Wegscheider, W.; Strunk, C. Phys. Rev. B: Condens. Matter Mater. Phys. 2007, 75, No. 033315

(5) Holleitner, A. W.; Blick, R. H.; Hüttel, A. K.; Eberl, K.; Kotthaus, J. P. Science 2002, 297, 70.

(6) Holleitner, A. W.; Blick, R. H.; Qin, H.; Hüttel, A. K.; Eberl, K.; Kotthaus, J. P. Phys. Rev. Lett. 2001, 87, 256802

(7) Folk, J. A.; Potok, R. M.; Marcus, C. M.; Umansky, V. Science 2003 $299,679$.

(8) Hanson, R.; Kouwenhoven, L. P.; Petta, J. R.; Tarucha, S.; Vandersypen, L. M. K. Rev. Mod. Phys. 2007, 79, 1217.

(9) Gustavsson, S.; Leturcq, R.; Studer, M.; Ihn, Th.; Ensslin, K. Driscoll, D. C.; Gossard, A. C. Nano Lett. 2008, 8, 2547.

(10) Bird, J. P.; Ochiai, Y. Science 2004, 303, 1621.
(11) Fischer, S. F.; Apetrii, G.; Kunze, U.; Schuh, D.; Abstreiter, G. Nature Phys. 2006, 2, 91.

(12) Rokhinson, L. P.; Larkina, V.; Lyanda-Geller, Y. B.; Pfeiffer, L. N.; West, K. W. Phys. Rev. Lett. 2004, 93, 146601.

(13) Topinka, M. A.; LeRoy, B. J.; Shaw, S. E. J.; Heller, E. J.; Westervelt, R. M.; Maranowski, K. D.; Gossard, A. C. Science 2000, 289, 2323.

(14) Topinka, M. A.; LeRoy, B. J.; Westervelt, R. M.; Shaw, S. E. J.; Fleischmann, R.; Heller, E. J.; Maranowski, K. D.; Gossard, A. C. Nature 2001, 410, 183.

(15) Jura, M. P.; Topinka, M. A.; Urban, L.; Yazdani, A.; Shtrikman, H.; Pfeiffer, L. N.; West, K. W.; Goldhaber-Gordon, D. Nature Phys. 2007, 3, 841

(16) Wilson, T.; Gannaway, J. N.; Johnson, P. J. Microsc. (Oxford, U. K.) 1980, 118, 309.

(17) Marcus, C.; Rimberg, A. J.; Westervelt, R. M.; Hopkins, P. R.; Gossard, A. C. Phys. Rev. Lett. 1992, 69, 506

(18) Dupont, E.; Corkum, P. B.; Liu, H. C.; Buchanan, M.; Wasilewski, Z. R. Phys. Rev. Lett. 1995, 74, 3596.

(19) Atanasov, R.; Haché, A.; Hughes, J. L. P.; van Driel, H. M.; Sipe, J. E. Phys. Rev. Lett. 1996, 76, 1703.

(20) Haché, A.; Kostoulas, Y.; Atanasov, R.; Hughes, J. L. P.; Sipe, J. E.; van Driel, H. M. Phys. Rev. Lett. 1997, 78, 306

(21) Hof, K.-D.; Rossler, C.; Manus, S.; Kotthaus, J. P.; Holleitner, A.; Schuh, D.; Wegscheider, W. Phys. Rev. B: Condens. Matter Mater. Phys. 2008, 78, 115325 .

(22) Rossler, C.; Hof, K.-D.; Manus, S.; Ludwig, S.; Kotthaus, J. P.; Simon, J.; Holleitner, A. W.; Schuh, D.; Wegscheider, W. Appl. Phys. Lett. 2008, 93, No. 071107.

(23) Thunich, S.; Prechtel, L.; Spirkoska, D.; Abstreiter, G.; Fontcuberta i Morral, A.; Holleitner, A. W. Appl. Phys. Lett. 2009, 95, No. 083111

(24) Laikhtman, B.; Sivan, U.; Yacoby, A.; Umbach, C. P.; Heiblum, M.; Kash, J. A.; Shtrikman, H. Phys. Rev. Lett. 1990, 65, 2181.

(25) Chaplik, A. V. Soviet Phys. JETP 1971, 33, 997.

(26) Giulani, G. F.; Quinn, J. J. Phys. Rev. B: Condens. Matter Mater. Phys. 1982, 26, 4421 .

(27) Schäpers, Th.; Krüger, M.; Appenzeller, J.; Förster, A.; Lengeler, B.; Lüth, H. Appl. Phys. Lett. 1995, 66, 3603.

(28) Cumming, D. R. S.: Davies, J. H. Appl. Phys. Lett. 1996, 69, 3363

(29) Snoke, D. W.; Ruhle, W. W.; Lu, Y.-C.; Bauser, E. Phys. Rev. B. Condens. Matter Mater. Phys. 1992, 45, 10979.

(30) Beenakker, C. W. J.; van Houten, H. Solid State Phys. 1991, 44, 1. 